\title{
Compatibility, Incompatibility, Tree-Width, and Forbidden Phylogenetic Minors
}

\author{
Sudheer Vakati ${ }^{1,3}$ \\ Amazon.com \\ Seattle, WA, USA \\ David Fernández Baca ${ }^{2,4}$ \\ Department of Computer Science \\ Iowa State University \\ Ames, IA 50010, USA
}

\begin{abstract}
A collection $\mathcal{P}$ of phylogenetic trees is compatible if there is a tree that displays all the relationships among species exhibited by the trees in $\mathcal{P}$. We give a simple characterization of compatibility based on graph triangulation. We then study how to deal with incompatibility through edge contraction and tree deletion, and introduce the notion of a phylogenetic minor.
\end{abstract}

Keywords: Triangulations, Treewidth, Phylogenetics

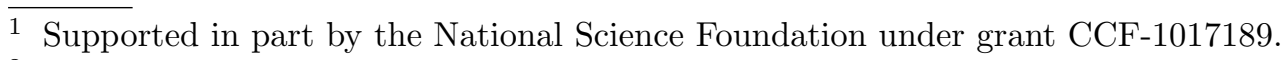

2 Supported in part by the National Science Foundation under grants CCF-1017189 and CCF-1422134.

3 Email: svakati@amazon.com

4 Email: fernande@iastate.edu 


\section{Introduction}

A phylogenetic tree $T$ is an unrooted tree with no degree two-vertices, whose leaves are in one-to-one correspondence with a label set $\mathcal{L}(T)$. The labels represent species - also known as taxa-, and $T$ represents the evolutionary history of these species. The following problem arises when constructing phylogenetic trees for a collection of species. We are given a collection of input trees with partially overlapping leaf sets. The question is to determine if there is a tree that exhibits the information from each of them - i.e., whether or not the information present in the input trees is compatible. To explain this precisely, we need some definitions.

Given a set $Y \subseteq \mathcal{L}(T)$, let $T \mid Y$ denote the tree obtained by suppressing vertices of degree two in the minimal subtree of $T$ connecting the leaves with labels in $Y$. Let $S$ be another phylogenetic tree with label set $\mathcal{L}(S) \supseteq \mathcal{L}(T)$. Then, $S$ displays $T$ if $T$ can be derived from $S \mid \mathcal{L}(T)$ by edge contraction.

Throughout the rest of the paper, $\mathcal{P}=\left\{T_{1}, T_{2}, \ldots, T_{k}\right\}$ denotes a collection of $k$ phylogenetic trees; we refer to $\mathcal{P}$ as a profile. We write $\mathcal{L}(\mathcal{P})$ to denote $\bigcup_{i \in[k]} \mathcal{L}\left(T_{i}\right)$. A supertree for $\mathcal{P}$ is a phylogenetic tree $S$ with label set $\mathcal{L}(S)=\mathcal{L}(\mathcal{P}) . \quad \mathcal{P}$ is compatible if there exists a supertree of $\mathcal{P}$ that displays every tree in $\mathcal{P}$. Deciding whether a profile is compatible is called the tree compatibility problem. While this problem is NP-Complete [5], it is fixed-parameter tractable in the number of trees [3].

\section{Display graphs, modified display graphs, and tree-width}

The display graph of $\mathcal{P}$ is the graph $G(\mathcal{P})$ obtained from the disjoint union of the trees in $\mathcal{P}$ by identifying the leaves with the same labels [3]. The modified display graph of $\mathcal{P}$, denoted $G_{\mathrm{M}}(\mathcal{P})$, is the graph obtained from $G(\mathcal{P})$ by doing the following for each leaf $v$ in $G(\mathcal{P})$ : (i) insert edges to make the neighbors of $v$ a clique and (ii) delete $v$. We refer to the edges inserted in step (i) as added edges; every other edge of $G_{\mathrm{M}}(\mathcal{P})$ is a tree edge. See Fig. 1. Unlike $G(\mathcal{P})$, none of the vertices of $G_{\mathrm{M}}(\mathcal{P})$ is labeled. Thus, two profiles with different display graphs and different label sets may have isomorphic modified display graphs, where the isomorphism distinguishes tree edges from added edges.

A tree decomposition of a graph $G$ is a pair $(T, B)$ where $T$ is a tree and $B$ is a function from $V(T)$ to subsets of $V(G)$ such that: (i) for every $v \in V(G)$, there is an $x \in V(T)$ where $v \in B(x)$, (ii) for every edge $u v \in E(G)$, there is an $x \in V(T)$ where $\{u, v\} \subseteq B(x)$, and (iii) for any $v \in V(G)$, the set $\{x: v \in B(x)\}$ induces a subtree in $T$. The width of a tree decomposition $(T, B)$ of $G$ is the maximum value of $|B(x)|-1$ over all vertices $x \in V(T)$. The 


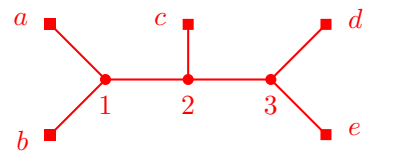

(i)

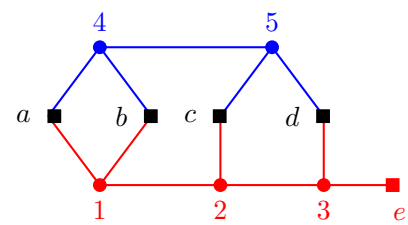

(iii)

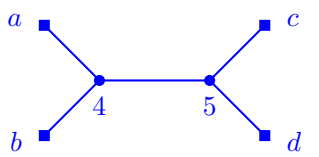

(ii)

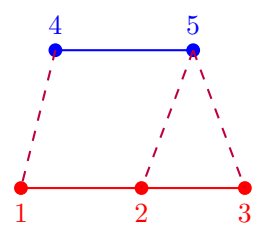

(iv)

Fig. 1. (i) and (ii) Input trees $T_{1}$ and $T_{2}$. (iii) Display graph of profile $\mathcal{P}=\left\{T_{1}, T_{2}\right\}$. (iv) Modified display graph of $\mathcal{P}$. Tree edges are solid; added edges are dashed.

treewidth of $G$, denoted by $\operatorname{tw}(G)$, is the smallest width of a tree decomposition of $G$. It is known that if profile $\mathcal{P}$ is compatible, $\operatorname{tw}(G(\mathcal{P})) \leq k[3]$.

\section{Characterizing Compatibility}

Let $G$ be a graph. Let $V(G)$ and $E(G)$ denote, respectively, the vertex and edge sets of $G$. A chord in a cycle $C$ in $G$ is an edge between two non-adjacent vertices of $C$. $G$ is chordal if and only if every cycle of length greater than three has a chord. A triangulation of $G$ is a chordal graph $H$ where $V(H)=V(G)$ and $E(G) \subseteq E(H)$.

In [7], we proved a characterization of compatibility based on triangulations. Next, we give a simpler characterization, based on the modified display graph $G_{\mathrm{M}}(\mathcal{P})$. Let us call a triangulation $H$ of $G_{\mathrm{M}}(\mathcal{P})$ legal if every clique in $H$ contains at most one tree edge.

Theorem 2.1 $\mathcal{P}$ is compatible if and only if $G_{\mathrm{M}}(\mathcal{P})$ has a legal triangulation.

It is well-known that tree compatibility can be tested in polynomial time when there is a label that is common to all the input trees $[1,5]$. The following corollary of Theorem 2.1 extends this observation to a larger class of profiles, where there is no such common label.

Corollary 2.2 If $G_{\mathrm{M}}(\mathcal{P})$ contains a $k$-clique with only added edges, then the compatibility of $\mathcal{P}$ can be determined in polynomial time.

The corollary follows from the observation that if $G_{\mathrm{M}}(\mathcal{P})$ contains a $k$ clique with only added edges, then there exists a profile $\mathcal{P}^{\prime}$ where all trees have a common label, such that $G_{\mathrm{M}}\left(\mathcal{P}^{\prime}\right)$ is isomorphic to $G_{\mathrm{M}}(\mathcal{P})$. See Fig. 2. 


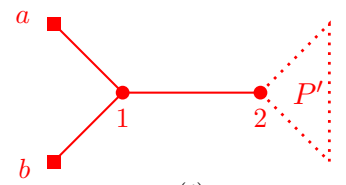

(i)

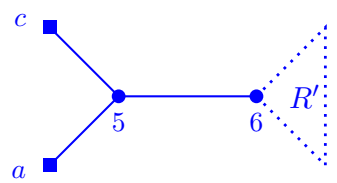

(iii)

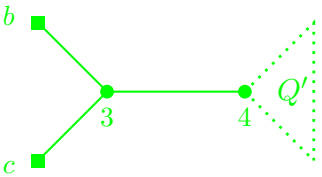

(ii)

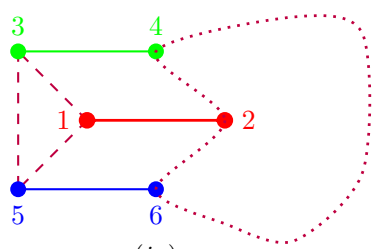

(iv)

Fig. 2. (i), (ii), and (iii) : Input trees $T_{1}, T_{2}$, and $T_{3}$, where $\mathcal{L}\left(T_{1}\right) \cap \mathcal{L}\left(T_{2}\right) \cap \mathcal{L}\left(T_{3}\right)=\emptyset$. (iv) $G_{\mathrm{M}}(\mathcal{P})$ for $\mathcal{P}=\left\{T_{1}, T_{2}, T_{3}\right\}$. Dotted lines represent potential paths.

\section{Edge Contraction and Tree Removal Problems}

Due to phenomena such as gene duplication and loss and lateral gene transfer, incompatible profiles are common in practice. We consider two approaches to deal with an incompatible profile $\mathcal{P}$. Let $p$ be a nonnegative integer. The edge contraction problem asks whether $\mathcal{P}$ can be made compatible by contracting at most $p$ internal edges. Note that it is always possible to make $\mathcal{P}$ compatible by contracting sufficiently many edges. The tree removal problem asks whether $\mathcal{P}$ can be made compatible by removing at most $p$ trees. The edge contraction and tree removal problems are NP-hard even for $p=0$. Next, we characterize the sets of trees that must be removed and the sets of edges that need to be contracted in order to achieve compatibility. Our results can be interpreted as counterparts to a result by Bordewich et al. [2] on the character removal problem.

A triangulation $H$ of $G_{\mathrm{M}}(\mathcal{P})$ is good if for every clique $K$ in $H$, and for every $i \in[k], V\left(T_{i}\right) \cap K$ induces a subtree in $T_{i}$. Note that every legal triangulation of $G_{\mathrm{M}}(\mathcal{P})$ is good, but the converse is not always true. For the next set of results, let $H$ be any good triangulation of $G_{\mathrm{M}}(\mathcal{P})$.

Let us first consider the edge contraction problem. A set of blocking edges for $H$ is a subset $Q$ of the tree edges of $G_{\mathrm{M}}(\mathcal{P})$ such that, for each maximal clique $K$ in $H, E(K) \backslash Q$ has at most one tree edge.

Theorem $3.1 \mathcal{P}$ can be made compatible by contracting at most $p$ internal edges if and only if $G_{\mathrm{M}}(\mathcal{P})$ has a good triangulation with at most $p$ blocking edges.

Corollary 3.2 If profile $\mathcal{P}$ can be made compatible by contracting at most $p$ 
internal edges, then $\operatorname{tw}\left(G_{\mathrm{M}}(\mathcal{P})\right) \leq k+p$.

We now consider the tree deletion problem. A set of blocking trees for $H$ is a subset $\mathcal{Y} \subseteq \mathcal{P}$ with the following property. Let $F$ be the set of all internal edges of trees in $\mathcal{P} \backslash \mathcal{Y}$. Then, for every maximal clique $K$ in $H, K$ contains at most one edge of $F$.

Theorem $3.3 \mathcal{P}$ can be made compatible by removing at most $p$ input trees if and only if $G_{\mathrm{M}}(\mathcal{P})$ has a good triangulation with at most $p$ blocking trees.

\section{Phylogenetic minors}

A minor of an undirected graph $G$ is a graph that can be obtained from $G$ by deleting vertices and edges and by contracting edges. A well-known theorem by Robertson and Seymour [4] states that every family of graphs that is closed under minors - in particular, graphs of bounded tree-width - can be defined by a finite set of forbidden minors. An interesting question is whether a similar characterization can be made for tree compatibility via modified display graphs. Indeed, the existence of small obstructions to compatibility would have important consequences for dealing with incompatibility via edge contraction or tree deletion, as it would enable us to isolate the sources of conflict to small sets of edges or trees.

As mentioned at the end of Section 1, a necessary condition for profile $\mathcal{P}$ to be compatible is that $\operatorname{tw}(G(\mathcal{P})) \leq k$, which implies that $\operatorname{tw}\left(G_{\mathrm{M}}(\mathcal{P})\right) \leq k$. This condition is also sufficient for $k=2$, but not for $k \geq 3$ [6]. Nevertheless, the following result implies that small obstructions to compatibility exist in the special case where all trees have the same leaf set; the result also yields an alternate proof of the tractability of instances of this kind.

Theorem 4.1 Suppose that $\mathcal{L}(T)=\mathcal{L}\left(T^{\prime}\right)$ for every two trees $T, T^{\prime} \in \mathcal{P}$. Then, $\mathcal{P}$ is compatible if and only if $\operatorname{tw}\left(G_{\mathrm{M}}(\mathcal{P})\right) \leq k$.

Proving a forbidden substructure characterization for profiles where not all trees have the same leaf set requires adapting the idea of a minor to phylogenetics. Consider the following approach. A phylogenetic minor of $G_{\mathrm{M}}(\mathcal{P})$ is a graph $G^{\prime}$ derived from $G_{\mathrm{M}}(\mathcal{P})$ by zero or more of the following operations: (1) contracting a tree edge, (2) deleting a maximal set of vertices $U$ such that the subgraph of $G_{\mathrm{M}}(\mathcal{P})$ induced by $U$ is a tree that contains no added edge, and (3) deleting an added edge. Operation (1) was considered in the previous section. So was (2): the operation is equivalent to deleting an input tree from $\mathcal{P}$. Operation (3) corresponds to renaming or deleting certain labels in $\mathcal{P}$. 
Lemma 4.2 If $G^{\prime}$ is a phylogenetic minor of $G_{\mathrm{M}}(\mathcal{P})$, then $G^{\prime}=G_{\mathrm{M}}\left(\mathcal{P}^{\prime}\right)$ for some profile $\mathcal{P}^{\prime}$ with at most $k$ trees.

Theorem 4.3 If $G_{\mathrm{M}}(\mathcal{P})$ has a legal triangulation, then so does any phylogenetic minor of $G_{\mathrm{M}}(\mathcal{P})$.

Several open questions remain. We know, for instance, that the forbidden minors for tree-width $k$ correspond to forbidden phylogenetic minors for the modified display graph of a compatible profile, but these are not the only forbidden phylogenetic minors. In one special case, however, they are.

Lemma 4.4 A profile $\mathcal{P}$ with $k=2$ phylogenetic trees is incompatible if and only if $G_{\mathrm{M}}(\mathcal{P})$ has a $K_{4}$ with two non-adjacent tree edges as a phylogenetic minor.

For $k=3$, we have identified a small set of forbidden phylogenetic minors that have tree-width less than $k$. We conjecture that these are the only such configurations.

\section{References}

[1] A. V. Aho, Y. Sagiv, T. G. Szymanski, J. D. Ullman, Inferring a tree from lowest common ancestors with an application to the optimization of relational expressions, SIAM J. Comput. 10 (3) (1981) 405-421.

[2] M. Bordewich, K. T. Huber, C. Semple, Identifying phylogenetic trees, Discrete Mathematics 300 (1-3) (2005) 30-43.

[3] D. Bryant, J. Lagergren, Compatibility of unrooted phylogenetic trees is FPT, Theor. Comput. Sci. 351 (2006) 296-302.

[4] N. Robertson, P. D. Seymour, Graph minors. XX. Wagner's conjecture, Journal of Combinatorial Theory, Series B 92 (2) (2004) 325-357.

[5] M. A. Steel, The complexity of reconstructing trees from qualitative characters and subtrees, Journal of Classification 9 (1992) 91-116.

[6] S. Vakati, "Fixed parameter algorithms for compatible and agreement supertree problems," Ph.D. thesis, Iowa State University (2013).

[7] S. Vakati, D. Fernández-Baca, Graph triangulations and the compatibility of unrooted phylogenetic trees, Appl. Math. Lett. 24 (5) (2011) 719-723. 